\title{
On Composite Service Optimization Across Distributed QoS Registries*
}

\author{
Fei Li, Fangchun Yang, Kai Shuang, and Sen Su \\ State Key Lab. of Networking and Switching, Beijing University of Posts and \\ Telecommunications \\ 187\#,10 Xi Tu Cheng Rd.,Beijing,100876, P.R. China \\ pathos.lf@gmail.com, \{shuangk, fcyang, susen\}@bupt.edu.cn
}

\begin{abstract}
Web service composition is a promising technology to effectively integrate distributed autonomous services in service oriented paradigm. When providing composite services, ensuring user experienced QoS (Quality of Service) in dynamic environment poses a great challenge. In this paper, we present a distributed service selection approach for optimizing composite service with complex structures. The approach does not require a centralized QoS registry to have complete composition logic, but runs on our distributed QoS architecture iteratively. Experimental results show that the approach is efficient and effective for our problem.
\end{abstract}

\section{Introduction}

Web service, as an implementation of service oriented architecture, is gaining more and more acceptance in both academia and industries. In web service framework, a set of XML(eXtensible Markup Language) based standards[1], greatly improves the interoperability of business applications. Services (In this paper, we use the term service and web service interchangeably) could publish their functional and nonfunctional attributes. Service users could automatically discover services. Service provider could integrate other provider's services to fulfill complex user requirements. The integration process is known as service composition and the integrated service is composite service.

Automatic service composition is a hot topic in web service research which aims easily reusing of business applications and fast provisioning of new services. S. Dustar and W. Schreiner[2] surveyed some of the important works on different aspects of service composition. Most of the early works are function related, as automatic generation of composition logic, or coordination of composite service. But user requirements are not only functional, also non-functional. Thus the "QoS driven service composition" problem was presented afterwards[3]. As far as we know, all

This work is supported by the National Basic Researchand Development Program (973 program) of China under Grant No.2003CB314806; the Program for New Century Excellent Talents in University (No:NCET-05-0114); the Program for Changjiang Scholars and Innovative Research Team in University (PCSIRT); the Hi-Tech Research and Development Program (863 Program) of China under Grant No.2006AA01Z164. 
published works was focusing on improving the selection algorithm performance and can only execute on a centralized entity called QoS center or registry. However, with the growing deployment of service oriented applications, centralized architectures may not satisfy the requirements of scalability and flexibility, and suffer from singlepoint failure. More importantly, for the requirements of global business, each centralized entity could only serve for a specific business region like a corporation or an organization, but not support global scale B2B(business-to-business) applications.

In this paper, we propose a distributed service selection approach based on our distributed QoS registry architecture[4]. The distributed QoS registry is a limited number of QoS registries which could communicate and cooperate with each other. Each one of them maintains QoS information of at least one set of functional identical services. These services could be selected based on their real-time QoS to optimize composite service in a dynamic environment. Figure 1 illustrates the scenario of a composite service logic and related distributed QoS registries. Every $\mathrm{Ti}$ is a set of services which could accomplish a specific task.

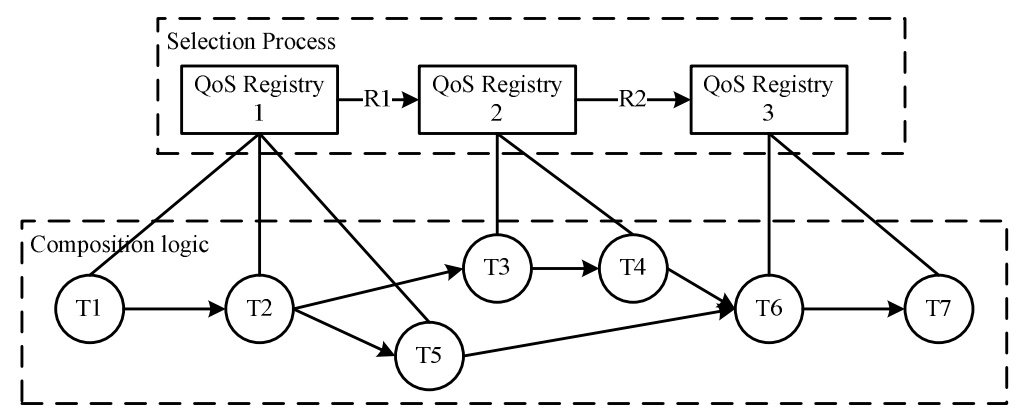

Fig. 1. Distributed QoS registries and a composite service

Our approach to solve the distributed service selection problem has two steps: first, we use an algorithm which could iteratively select services to optimize sequence structure. The algorithm is improved from our previous algorithm by using a more mature heuristic approach. Second, we divide a composite service to several connected sequence structures. The selection results for these structures are aggregated iteratively to optimize the whole composite service.

The rest of the paper is organized as follows: Section 2 reviews some related works. Section 3 describes the distributed selection algorithm for sequence structure in detail. Section 4 presents the approach to apply the algorithm on complex composite structure in distributed environment. Section 5 discusses some experimental results. Finally, the paper concludes in Section 6.

\section{Related Works}

Some pioneer works have been done on distributed service architecture. Distributed orchestration of web services has been discussed in Benatallah and Sheng et al.[5] for 
the first time. They designed the SELF-SERV (compoSing wEb accessibLe inFormation \& buSiness sERVices) platform for dynamic and peer-to-peer provisioning of web services. Composite service execution on the platform was in peer-to-peer manner without centralized coordinator. Chafle and Nanda et al.[6] researched some critical problems in automating decentralized execution of composite service. They provided a method for automatic BPEL code partition deriving from program partition method for multiprocessor execution.

A lot of works have been done on QoS based service selection for composite web service. Zeng et al.[3] has presented a basic QoS model for service composition which solved by Integer Programming. For the problem is NP-hard[7], many heuristic approaches have been carried out to improve efficiency. For example: Canfora et al.[7] proposed a genetic algorithm approach to optimize the selection process; Yu et al.[8] model the problem as a Multi-choice, Multi-dimension 0-1 Knapsack Problem (MMKP) and used a modified HEU algorithm to solve it.

In our previous work[4], we have presented a distributed QoS registry architecture and a QoS model with network condition. Based on our architecture and QoS model, we designed a distributed heuristic algorithm to optimize critical task path.

\section{Service Selection Algorithm for Sequence Structure}

For service selection problem in centralized environment, where QoS information of all candidate services is stored in one registry, the selection processes are not different for sequence structure and other structures. But in decentralized environment, we have to deal with them respectively because no registry has the whole composition logic. This section describes the selection algorithm for sequence structure.

\subsection{Problem Definition}

A sequence structure with $l$ tasks is $p=\left\langle t_{1}, t_{2}, \ldots, t_{l}\right\rangle$, where $t_{i}$ is the $i$ th task in topological order. Each task has a set of candidate services, $S(t)=\left\{s_{1}, s_{2}, \ldots, s_{m}\right\}$, one of them will be selected for corresponding task. Each service $s$ has a set of QoS parameters $Q(s)=\left\langle q_{1}, q_{2}, \ldots, q_{n}\right\rangle$ monitored by QoS registry. The composite service $c s$ also has a set of QoS parameters $Q(c s)=\left\langle q_{1}^{c}, q_{2}^{c}, \ldots, q_{n}^{c}\right\rangle$, where $q_{i}^{c}=f_{i}\left(q_{i}^{1}, q_{i}^{2}, \ldots, q_{i}^{m}\right), 1 \leq i \leq n, f_{i}$ computes the compositional effects of the $i$ th QoS parameter. User could give constraints for composite service on any parameter $C=\left\langle c_{1}, c_{2}, \ldots, c_{n}\right\rangle$. Our target is to optimize $Q(c s)$ and make sure no QoS parameter exceeds the constraints $C$. Every possible composition of services is called a plan. We use the common accepted weighted average to evaluate a plan. In this paper, we assume the better plan is the one with smaller score. $w_{i}$ is the significance of the $i$ th QoS parameter:

$$
\operatorname{Score}(p)=\sum_{1 \leq i \leq z} w_{i} q_{i}^{p},\left(0 \leq w_{i} \leq 1, \sum_{1 \leq i \leq z} w_{i}=1\right)
$$

This is a common optimization problem but no existing approaches are applicable to our case, because in our selection model, any registry may only have a part of the 
sequence. We need an iterative algorithm which could be applied to tasks one by one and finally get global optimized QoS. For space reasons, we only describe idea of the algorithm and a further optimization in this paper. Interested reader can refer to our previous publication[4] for more details.

\subsection{Iterative Selection Algorithm}

The basic ISA is enlightened by Extended Bellman-Ford Algorithm (EBFA)[9], but we modified it for optimizing nodes QoS rather than link QoS. For each task, basic ISA computes scores of all possible plans with previous result and records new plans in each service node for next computing iteration. If currently computing task is $t_{i}(1<i \leq l)$, the algorithm should have all possible plans from $t_{1}$ to $t_{i-1}$ and carry out plans from $t_{1}$ to $t_{i}$. When the last task is computed, the best plan is selected.

Basic ISA has a significant problem. With the growing of candidate service number, the recorded plan number grows exponentially. For a task path with $l$ tasks and $m$ services for each task, the candidate plans would be $m^{l}$. In practice, services with better scores have higher possibility to be selected in the final composition plan. We have proposed a heuristic algorithm in our previous work which keeps $K$ plans with the best scores in each service node, called ISA-Heu here. The algorithm can be further optimized.

The best plan for a part of the sequence structure may not always be the best for the whole sequence. In computation, some of the plans' QoS parameter may be too close to constraints, even if they have excellent score, they are highly likely to exceed constraints in next iteration. Task optimization should take user constraints into account and predict which plan is better for overall composite service. We achieve this prediction by adjust the scoring function applied in middle task computation. The new scoring function could magnify the effects of QoS parameters which are approaching constraints. We call this algorithm ISA-HeuPred. The adjusted scoring function with prediction is:

$$
\operatorname{Score} \operatorname{Pr} e d(p)=\sum_{1 \leq i \leq n} w_{i} \frac{q_{i}^{s}}{c_{i}-q_{i}^{s}},\left(0 \leq w_{i} \leq 1, \sum_{1 \leq i \leq n} w_{i}=1, q_{i}^{s}<c_{i}\right)
$$

Suppose there are $l$ tasks in critical task path and each task has $m$ candidate services, in the worst case, the time complexity of ISA is $O\left(m^{l}\right)$, otherwise, the time complexity of ISA-Heu and ISA-HeuPred is $O\left(K^{2} l m^{2}\right)$. The space complexity of ISA-Heu and ISA-HeuPred is $O(\mathrm{Km})$.

\section{Iterative Computing for Composition Structures}

Currently, mainstream composition logic description approach as WSBPEL (Web Services Business Process Execution Language) [10] is developed from traditional 
business process modeling works. Sequence, switch, parallel, loop and pick are familiar basic structures for constructing composite service. In our distributed QoS architecture, no QoS registry has an overall view of the whole composition logic, even these basic structures may not exist on a single registry, so the QoS optimization of whole composite service have to be carried out in distributed and iterative manner.

Although registries have no knowledge of the overall process, they have the information that a specific task has how many predecessors and successors. For a task node has more than one successor, as a parallel, switch or pick begins from the node, we call it a branch node. For a task node has more than one predecessors, we call it an aggregation node. Both branch node and aggregation node are structure nodes. Other nodes are all called sequence nodes. For example, in Fig.1, T2 is a branch node, T6 is an aggregation node. For the node classification, 2 issues should be noticed: 1, loop structure could be transformed to limited number of switch structures[3][11]; 2, when partitioning, it does not matter what kind of branch it is, the branch type only affects QoS aggregation.

We define a task path as a set of task nodes begin at the start task node or a structure node, and end at the final task node, or before the next structure node in topology order. By this definition, a composition logic could be divided into several connected task path where every path is a sequence structure. In Fig.1, there are 4 task paths: $p_{1}=<t_{1}>, p_{2}=<t_{2}, t_{3}, t_{4}>, p_{3}=<t_{2}, t_{5}>, p_{4}=<t_{6}, t_{7}>$. These task paths are computed iteratively in topological order. When the branch node sends result to next task paths, the branch type (parallel, switch or pick) and node identification is sent with the result, so that corresponding aggregation node could compute aggregated QoS of previous paths. As presented in [3], every QoS parameter has an aggregation function. For example, price is the summary of all the selected services' price, availability is the product of all the selected services' availability. For service selection before composite service running, the aggregation functions are different for different structures. The detailed aggregation method of QoS parameter is not in the scope of this paper, but it is obvious that the optimal result of task paths aggregated together is still optimal.

The overall selection process for composite service in Fig.1 by our approach is: compute $p_{1}$ at registry 1 first. Based on $p_{1}$ 's result, compute $p_{2}$ at registry 1 and $p_{3}$ at registry 2 respectively. In fact, we optimize $p_{1}+p_{2}=<t_{1}, t_{2}, t_{3}, t_{4}>$ and $p_{1}+p_{3}=<t_{1}, t_{2}, t_{5}>$ as 2 independent sequences. $p_{1}, p_{2}$, and $p_{3}$ are aggregated at registry 3 . Here, registry 3 identifies results of $p_{2}$ and $p_{3}$, aggregates them based on branch type, then combines with $p_{1}$. From the viewpoint of $t_{7}$, the topology before $t_{6}$ has nothing to do with its computation. Then, registry 3 finish computation of $t_{7}$ based on aggregated result of $t_{6}$.

The aggregation operation would bring additional execution time to selection algorithm but it could be ignored. If $\mathrm{n}$ task nodes are aggregation nodes, the time complexity of using ISA-Heu for task path would be $O\left(K^{2} l m^{2}\right)+O(n)=O\left(K^{2} l m^{2}\right)$. 


\section{Experiments}

We studied the performance and effectiveness of our approach in different cases by a series of experiments. The experiments were conducted on a LINUX server with configuration of a Xeon $3.20 \mathrm{GHz}$ CPU and 2GB RAM, running Red Hat LINUX.

\subsection{Evaluation Methodology}

At first, we compare the performance of 2 types of ISA: non-heuristic and heuristic. In both cases, each service has 6 QoS parameters and each parameter is a randomly generated integer between 1 and 100. No constraints on any QoS parameter because we would like to test the worst case, but constraints could contribute to delete some plans and improve the execution time. For non-heuristic ISA, task number in composite service ranges from 1 to 10 and each task has 4,5 or 6 candidate services respectively. In heuristic case, for the scalability of ISA-Heu, task number in composite service ranges from 10 to 100 with a step of 10 and each task has 20,40 or 60 candidate services. Composite service is constructed by different structures, but these structures do not affect the overall selection time significantly, so we generate test composition logic by randomly repeating nodes in Fig.1. Aggregation function of every QoS parameter at $t_{6}$ is product. $K$ is set at 5 . The performance of ISA-Heu and ISA-HeuPred is the same so we only conduct our test on ISA-Heu. We run each case 100 times.

The heuristic algorithm may discard some "better" plans in computation. We study the effectiveness of 2 heuristic approaches by 2 criteria which has been used in our previous work: success ratio and approximation. For both heuristic approaches, we fix the task number at 8 and candidate service number for each task at 6 . The constraints is adjusted to make $90 \%$ cases could have at least one feasible plan. The heuristic parameter $K$ ranges from 1 to 8 .

\subsection{Result and Analysis}

Figure 2 shows the performance comparison of ISA and ISA-Heu. Because the heuristic approach limit plan number kept in each service node, the plan search time in each iteration is greatly decreased. When task number is 9 and 6 services for each task, the computation time of ISA approaches 10 seconds and grows exponentially. Otherwise, the ISA-Heu could easily scale up to 5000 candidate services and computation time is under 2 seconds. In practical composite service scale, ISA-Heu could complete in several milliseconds.

The effectiveness of ISA-Heu and ISA-HeuPred are illustrated in Fig. 3. When $\mathrm{K}<5$, the success ratio of ISA-HeuPred is much higher than ISA-Heu. Especially when $\mathrm{K}=1$, the heuristic with prediction could get a very impressive ratio at about $92 \%$. However, the approximation of ISA-HeuPred is lower than ISA-Heu by about 1 


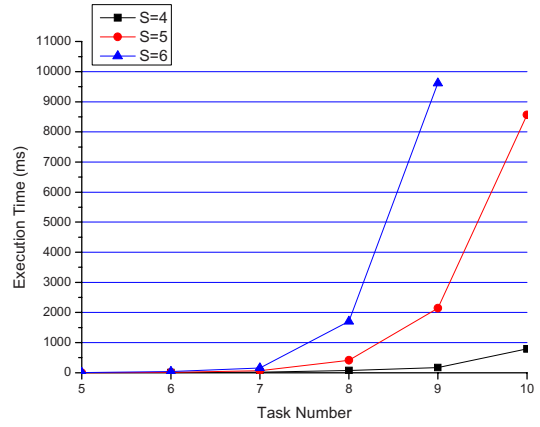

(a)

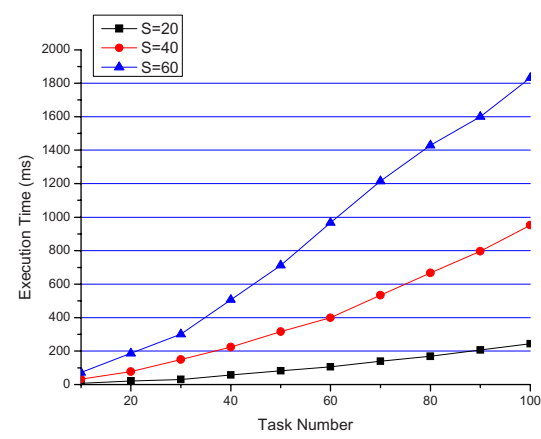

(b)

Fig. 2. (a) Execution time of ISA. (b) Execution time of ISA-Heu/ISA-HeuPred.

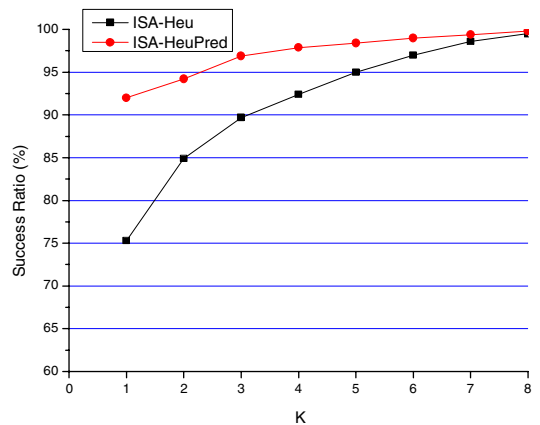

(a)

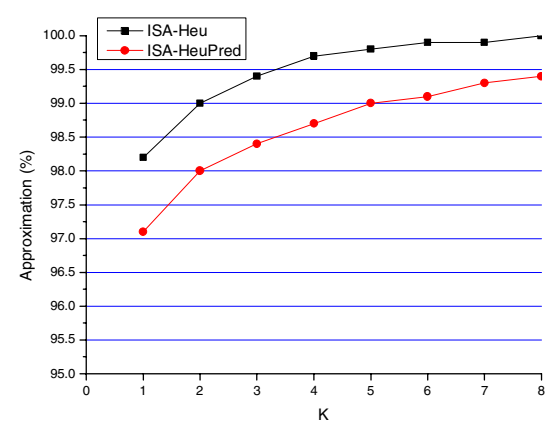

(b)

Fig. 3. (a) Success ratio of ISA-Heu and ISA-HeuPred. (b) Approximation of ISA-Heu and ISA-HeuPred.

percent in all tested values of $K$. But considering the approximation when $\mathrm{K}=1$ could exceed $97 \%$, this loss of approximation is highly acceptable.

\section{Conclusion}

In this paper, we presented a distributed service selection approach running on decentralized QoS registry architecture. The approach is combined by a service selection algorithm for simple sequence structure, and a method to divide complex structure to several connected sequence structures. The selection algorithm is modified from EBFA. By applying heuristic improvements which record the plans most likely to optimize overall path, the algorithm achieved excellent performance. The result of service selection for sequence structure could be aggregated at run-time to optimize the overall QoS of composite service. 


\section{Reference}

1. Tsalgatidou, A., Pilioura, T.: An Overview of Standards and Related Technology in Web Services. Distributed and Parallel Databases 12(2) (2002) 135-162

2. Dustdar, S., Schreiner, W.: A survey on web services composition. International Journal of Web and Grid Services 1(1) (2005) 1-30

3. Zeng, L., Benatallah, B., Ngu, A., Dumas, M., Kalagnanam, J., Chang, H.: QoS-Aware Middleware for Web Services Composition. IEEE Transactions on Software Engineering 30(5) (2004) 311-327

4. Li, F. Su, S., Yang, F.C.: On Distributed Service Selection for QoS Driven Service Composition. Proceedings of the 7th International Conference on Electronic Commerce and Web Technologies,EC-Web'06,LNCS 4082 (2006)

5. Benatallah, B., Dumas, M., Sheng, Q., Ngu, A.: Declarative composition and peer-to-peer provisioning of dynamic Web services. Proceedings of the $18^{\text {th }}$ International Conference on Data Engineering, ICDE'02 (2002) 297-308

6. Nanda, M., Chandra, S., Sarkar, V.: Decentralizing execution of composite web services. Proceedings of the 19th annual ACM SIGPLAN conference on Object-oriented programming, systems, languages, and applications, OOPSLA'04 (2004) 170-187

7. Canfora, G., Di Penta, M., Esposito, R., Villani, M.: An approach for QoS-aware service composition based on genetic algorithms. Proceedings of the Genetic and Evolutionary Computation Conference, GECOO'05 (2005) 1069-1075

8. Yu, T., Lin, K.: Service Selection Algorithms for Composing Complex Services with Multiple QoS Constraints. Proceedings of the 3rd International Conference on Service Oriented Computing, ICSOC'05, LNCS (3826) 130-143

9. Yuan, X.: On the extended Bellman-Ford algorithm to solve two-constrained quality of service routing problems. Proceedings of the 8th International Conference on Computer Communications and Networks, ICCCN'99 (1999) 304-310

10. OASIS, Web Services Business Process Execution Language Version 2.0 Public Review Draft, http://docs.oasis-open.org/wsbpel/2.0/, 23rd August, 2006

11. M. Gillmann, G. Weikum, and W. Wonner.: Workflow Management with Service Quality Guarantees. Proc. ACM SIGMOD Int'l Conf. Management of Data, pp. 228-239, June 2002. 